\title{
Restrictions and Generalizations on Comma-Free Codes
}

\author{
Alexander L. Churchill \\ Student \\ Stanford University, California, USA \\ achur@stanford.edu
}

Submitted: Feb 13, 2008; Accepted: Feb 14, 2009; Published: Feb 20, 2009

Mathematics Subject Classifications: 94B50, 94B65

\begin{abstract}
A significant sector of coding theory is that of comma-free coding; that is, codes which can be received without the need of a letter used for word separation. The major difficulty is in finding bounds on the maximum number of comma-free words which can inhabit a dictionary. We introduce a new class called a self-reflective comma-free dictionary and prove a series of bounds on the size of such a dictionary based upon word length and alphabet size. We also introduce other new classes such as self-swappable comma-free codes and comma-free codes in $q$ dimensions and prove preliminary bounds for these classes. Finally, we discuss the implications and applications of combining these original concepts, including their implications for the NP-complete Post Correspondence Problem.
\end{abstract}

\section{Introduction}

\subsection{Comma-free codes}

Comma-free codes were first introduced by Crick, Griffith, and Orgel [2] in 1957 as a potential explanation for the fact that DNA codes only twenty amino acids, despite the fact that it is a code with word-length three and a four-letter alphabet. While this explanation was revealed to be incorrect, comma-free codes are still a major area of exploration in coding theory. Initially, we establish definitions.

Let $n$ be a fixed positive integer. Consider a dictionary of words in which each word has length $k$ chosen from an $n$-letter alphabet. Let the alphabet consist of letters $a_{1}, a_{2}$, $a_{3}, \ldots, a_{n}$.

A set $D$ of $k$-letter words is called a Comma-Free Dictionary (according to Golomb, Gordon, and Welch [4]) if whenever words $a_{1} a_{2} \cdots a_{k}$ and $b_{1} b_{2} \cdots b_{k}$ are in $D$, the "overlaps" $a_{2} a_{3} \cdots a_{k} b_{1}, a_{3} \cdots a_{k} b_{1} b_{2}, \ldots, a_{k} b_{1} b_{2} \cdots b_{k-1}$ are not in $D$. 
The major problems investigated have been in determination of the maximum number of words a comma-free dictionary can possess, according to Levenshtein [6]. If the size of each word is $k$ and the size of the alphabet is $n$, the maximum number of elements in $D$ is denoted as $W(k, n)$. Golomb, Gordon, and Welch [4] established a bound for the maximum size of a comma-free dictionary as

$$
W(k, n) \leq \frac{1}{k} \sum_{d \mid k} \mu(d) n^{k / d},
$$

where $\mu(d)$ is the Möbius function. This bound is established by noticing several phenomena.

Initially, we consider equivalence classes of words formed by taking cyclic shifts of the letters of that word. We have equivalence classes $\bar{\omega}$ which contains all cyclic shifts $\phi^{i}(\omega)$. We define a cyclic shift $\phi^{i}(\omega)$ where $\phi\left(a_{1} a_{2} \cdots a_{k}\right)=a_{2} a_{3} \cdots a_{k} a_{1}$. For instance, ABCD and $\mathrm{CDAB}$ are cyclic shifts of each other, so they are in the same equivalence class. Furthermore, we observe that a comma-free dictionary cannot contain more than one member from each equivalence class. To show this, consider the overlaps formed by repeating one word in the equivalence class. This yields overlaps of all other words in the equivalence class. Repeating $A B C D$ gives ABCDABCD which contains $C D A B$ as an overlap.

Golomb, Gordon, and Welch [4] also put forth the concept of subperiod. Let $d$ be a divisor of $k$. We say that a word $a_{1} a_{2} \cdots a_{k}$ has subperiod $d$ if it is of the form $a_{1} a_{2} \cdots a_{d} a_{1} a_{2} \cdots a_{d} \cdots \cdots a_{1} a_{2} a_{d}$. If a word has subperiod $d<k$, such as ABCABC, it cannot be contained in a comma-free dictionary, because repeating such a word to yield $A B C A B C A B C A B C$ contains the original word as an overlap. We call a word with subperiod $d=k$ primitive.

The bound (1) is calculated by counting all equivalence classes with subperiod $k$. Golomb, Gordon, and Welch [4] provedthis bound was tight for $k=1,3,5,7,9,11,13$, and 15, and conjectured that it was tight for all odd $k$. This was proved by Eastman [3] in 1965. The only tight bound for even $k$ was given by Golomb, Gordon, and Welch [4]. They found that

$$
W(2, n) \leq\left\lfloor\frac{1}{3} n^{2}\right\rfloor
$$

Finding a general tight bound for all even $k$ is an open problem.

\section{Self-reflective comma-free codes}

One focus of this paper is Self-Reflective Comma-Free Codes. Initially, we must establish a definition. Let $\sigma\left(a_{1} a_{2} \cdots a_{k}\right)=a_{k} a_{k-1} \cdots a_{2} a_{1}$. We note that for every commafree dictionary $D=\left\{\omega_{1}, \omega_{2}, \ldots, \omega_{x}\right\}$, there is a similar comma-free dictionary $D=$ $\left\{\sigma\left(\omega_{1}\right), \sigma\left(\omega_{2}\right), \ldots, \sigma\left(\omega_{x}\right)\right\}$.

Definition: A set $D_{r} \subseteq D$ (where $D$ is a comma-free dictionary) is called a selfreflective comma-free dictionary if for all words $\omega \in D_{r}, \sigma(\omega) \in D_{r}$. The focus of this paper is to establish bounds on the maximum size of self-reflective comma-free dictionaries for general $n$ and $k$. Denote the greatest number of words $D_{r}$ can possess as $W_{r}(k, n)$. 


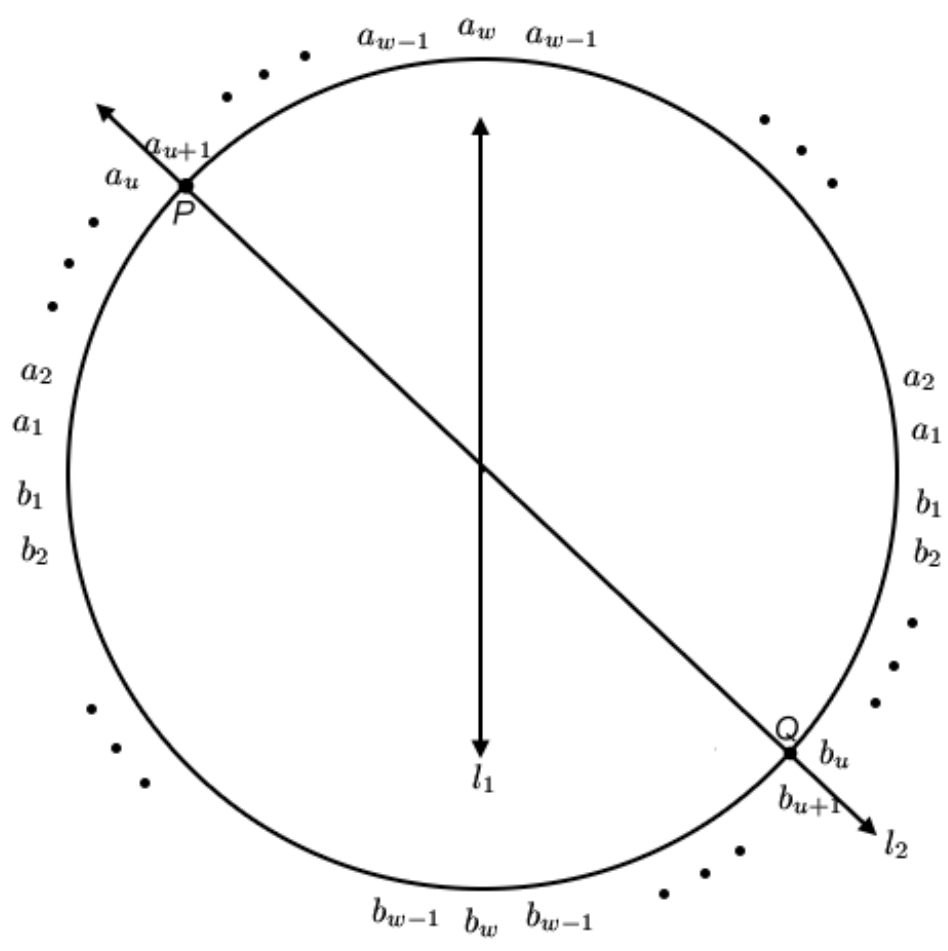

Figure 1: Bijective Circle

\section{$2.1 \quad$ Results}

\subsubsection{Lemmas}

We utilize the following lemmas for assistance in proving bounds on the size of selfreflective comma-free dictionaries. They give insight into word structure and properties of specific word types.

Lemma 1. If $\sigma\left(\omega_{1}\right) \in \overline{\omega_{1}}$, then $\sigma\left(\phi^{i}\left(\omega_{1}\right)\right) \in \overline{\omega_{1}}$ for all $i$.

Proof. When $i=0$, the proof is trivial. Assume $i>1$.

$$
\sigma\left(a_{i+1} a_{i+2} \cdots a_{k} a_{1} a_{2} \cdots a_{i-1} a_{i}\right)=a_{i} a_{i-1} \cdots a_{2} a_{1} a_{k} \cdots a_{i+2} a_{i+1},
$$

but we know $a_{i-1} a_{i-2} \cdots a_{2} a_{1} a_{k} \cdots a_{i+1} a_{i} \in \overline{\omega_{1}}$, so $a_{i} a_{i-1} \cdots a_{2} a_{1} a_{k} \cdots a_{i+2} a_{i+1} \in \overline{\omega_{1}}$.

This completes our proof.

Lemma 2. Let $\omega=a_{1} a_{2} \cdots a_{w-1} a_{w} a_{w-1} \cdots a_{2} a_{1} b_{1} b_{2} \cdots b_{w-1} b_{w} b_{w-1} \cdots b_{2} b_{1}$. If $\omega$ is primitive, then there does not exist any $\omega_{1}$ such that $\omega_{1} \in \bar{\omega}$ and $\sigma\left(\omega_{1}\right)=\omega_{1}$.

Proof. Assume some $\omega_{1}$ exists. Let $\omega_{1}=b_{u} b_{u-1} \cdots b_{1} a_{1} \cdots a_{w} \cdots a_{1} b_{1} \cdots b_{w} \cdots b_{u+1}$.

Consider a bijective circle in which each letter of $\omega_{1}$ is represented by a coloring of points around a circle, as shown in Figure 1. This figure, by construction, is fixed under reflection about $l_{1}=\overleftrightarrow{a_{w} b_{w}}$. Furthermore, we assume $\omega$ is self-reflective, so it must also 


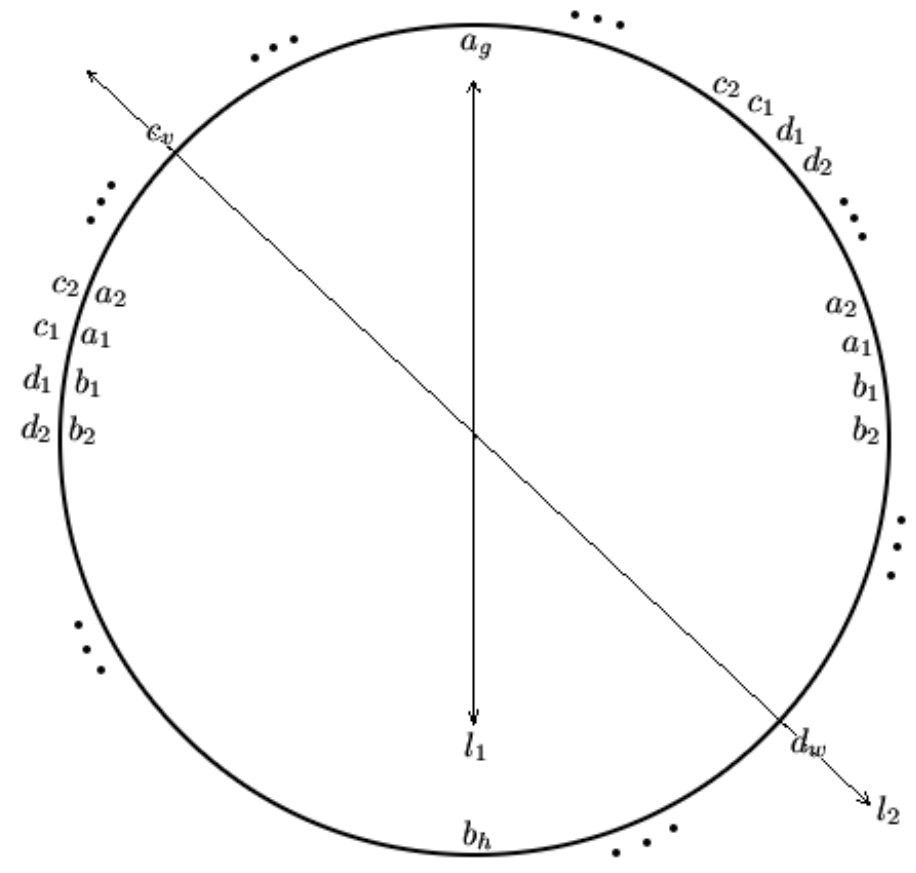

Figure 2: Bijective Circle

be fixed under reflection about $l_{2}=\overleftrightarrow{P Q}$ where $\mathrm{P}$ and $\mathrm{Q}$ are the midpoints of $\overline{a_{k} a_{k+1}}$ and $\overline{b_{k} b_{k+1}}$ respectively.

But since the circle-word is fixed under reflection about $l_{1}$ and $l_{2}$, where $l_{1} \neq l_{2}$, it is also fixed under the nonidentity rotation $l_{1} \circ l_{2}$. Since it is fixed under some nonidentity rotation, the word itself must be fixed under some cyclic shift $\phi^{i}(\omega)$ where $i \neq k$. But since it is fixed under some such cyclic shift, it must have some subperiod such that $d \mid k$ and $d \neq k$. Thus it is not primitive. This contradiction proves the lemma.

Lemma 3. Every word $\omega$ such that $\sigma(\omega) \in \bar{\omega}$ takes the form $\omega_{1} \omega_{2}$ where $\omega_{1}$ and $\omega_{2}$ are palindromes. Call such a word doubly palindromic.

Proof. Assume without loss of generality that $\omega=a_{1} a_{2} \cdots a_{k-1} a_{k}$ and let $\sigma(\omega)=a_{u} a_{u+1} \cdots a_{k-1} a_{k} a_{1} a_{2} \cdots a_{u-1}$.

But then $a_{u} a_{u+1} \cdots a_{k-1} a_{k} a_{1} a_{2} \cdots a_{u-2} a_{u-1}=a_{k} a_{k-1} \cdots a_{u+1} a_{u} a_{u-1} a_{u-2} \cdots a_{2} a_{1}$.

Clearly $a_{u} a_{u+1} \cdots a_{k-1} a_{k}$ and $a_{1} a_{2} \cdots a_{u-2} a_{u-1}$ are palindromes.

Thus, the word takes the desired form, which completes our proof.

Lemma 4. If $\omega_{1}=\omega_{2}$ where $\omega_{1}=a_{1} a_{2} a_{3} \cdots a_{g} \cdots a_{3} a_{2} a_{1} b_{1} b_{2} b_{3} \cdots b_{h} \cdots b_{3} b_{2} b_{1}$ and $\omega_{2}=c_{1} c_{2} c_{3} \cdots c_{v} \cdots c_{3} c_{2} c_{1} d_{1} d_{2} d_{3} \cdots d_{w} \cdots d_{3} d_{2} d_{1}$, then $\omega_{1}$ and $\omega_{2}$ have subperiod of length $\operatorname{gcd}(|i-j|, k)$ where $i=2 g-1$ and $j=2 v-1$.

Proof. Consider a bijective circle as in Lemma 2, shown in Figure 2. 
By construction, both words $\omega_{1}$ and $\omega_{2}$ are fixed under reflection about $l_{1}=\overline{a_{g} b_{n}}$ and $l_{2}=\overline{c_{v} d_{w}}$, so they are fixed about the rotation $l_{1} \circ l_{2}$ which rotates each letter by twice the angle of the intersection of $l_{1}$ and $l_{2}$. That is, each letter rotates by $2(g-v)=i-j$. Thus any two letters separated by $i-j$ will be equal. This rotation generates the same subgroup of $D_{k}$ as does rotation by $\operatorname{gcd}(|i-j|, k)$. Therefore the subperiod is of the desired length.

\subsubsection{Results for specific $k$}

Theorem 1. $W_{r}(2, n)=0$ for all $n$

Proof. We prove by contradiction. Assume $W_{r}(2, n)>0$. Let $F_{n}=a_{1} a_{2} \cdots a_{n}$ be an $n$-letter alphabet. Suppose there exists a word in our dictionary, $D_{r}$.

Without loss of generality, $\omega_{1} \in D_{r}$ where $\omega_{1}=a_{1} a_{2}$. Then $\sigma\left(\omega_{1}\right) \in D_{r}$ so $a_{2} a_{1} \in D_{r}$.

But $a_{2} a_{1}$ is a cyclic shift of $a_{1} a_{2}$ which cannot be part of a comma-free dictionary according to Crick, Griffith and Orgel [2]. This is a contradiction which completes our proof.

Theorem 2. $W_{r}(3, n) \leq \frac{2 n^{3}-3 n^{2}+n}{6}$;

Proof. We use bound (1) which counts the number of equivalence classes with subperiod $k$

$$
W(k, n) \leq \frac{1}{k} \sum_{d \mid k} \mu(d) n^{k / d} .
$$

This gives us $W(3, n) \leq \frac{1}{3}\left(n^{3}-n\right)$.

But this includes the equivalence classes $\overline{a b b}$ and $\overline{a b a}$. We cannot have both aba and bab in our comma-free dictionary, so for each pair of letters, there is either a counted word of the form abb or bba or of aab or baa. Without loss of generality, assume we have $\mathrm{abb}$ and bba. (In a self-reflective dictionary, both or neither must appear.) Since they are members of the same equivalence class, neither can appear, so we can subtract the equivalence class from our upper bound. There is one such equivalence class for every two letters which we can eliminate, for a total of $\left(\begin{array}{l}n \\ 2\end{array}\right)$ total. We subtract to get

$$
W_{r}(3, n) \leq \frac{2 n^{3}-3 n^{2}+n}{6}
$$

Theorem 3. $W_{r}(3, n)=\frac{2 n^{3}-3 n^{2}+n}{6}$.

Proof. We use the construction given by Crick, Griffith, and Orgel [2] for $n$ letters, removing those of the form ABB. Use the numbers 1 through $n$ to represent an $n$-letter comma-free alphabet, giving a well-ordered set. In this description, $A B{ }_{B}^{A}$ represents $\mathrm{ABA}$ and $\mathrm{ABB}$. 


$$
\begin{aligned}
& \begin{array}{ll}
1 & 1 \\
2 & 2 \\
3 & 3
\end{array} \\
& 121
\end{aligned}
$$

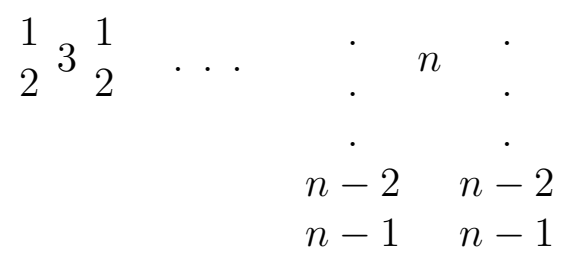

This is a comma-free code which has $1^{2}+2^{2}+3^{2}+\cdots n^{2}=\frac{2 n^{3}+3 n^{2}+n}{6}$ members. It is also self-reflective, because for all words $a b c, c b a$ must also be a member. This proves the bound from Theorem 2 is tight.

\subsubsection{Results for $k$ odd}

Theorem 4. For odd $k, W_{r}(k, n) \leq \frac{1}{k} \sum_{d \mid k} \mu(d) n^{k / d}-\left(\begin{array}{l}n \\ 2\end{array}\right)$.

Proof. Consider equivalence classes $\overline{a b a b a b \cdots a b a}$ and $\overline{b b a b a b a \cdots b a}$. Take words $\omega_{1}$ and $\omega_{2}$ in our dictionary from each respective equivalence class. Both $\sigma\left(\omega_{1}\right)=\omega_{1}$ and $\sigma\left(\omega_{2}\right)=\omega_{2}$ cannot be true. This is because then both abab $\cdots$ aba and baba $\cdots$ bab would necessarily be $\omega_{1}$ and $\omega_{2}$. This is not comma-free, because (abab $\cdots$ aba) (baba $\cdots$ bab) would then have $\omega_{1}$ and $\omega_{2}$ as an overlap. Thus, at least one word from one of the two equivalence classes must not reflect to itself. However, a reflection of either one of the equivalence classes yields a cyclic shift of that equivalence class, which is not allowed in a comma-free dictionary. Thus we subtract at least one of these two equivalence classes from bound (1). We subtract an equivalence class for each two letters, so there are a total of $\left(\begin{array}{l}n \\ 2\end{array}\right)$ eliminated, giving us our desired bound.

\subsubsection{Results for $k$ even}

Theorem 5. For $k=2(\bmod 4)$,

$$
W_{r}(k, n) \leq \frac{1}{k} \sum_{d \mid k} \mu(d) n^{k / d}-\left(\begin{array}{c}
n^{(k+2) / 4} \\
2
\end{array}\right)+\sum_{d \mid \frac{k}{2}, d \neq \frac{k}{2}}\left(\begin{array}{c}
n^{(d+1) / 2} \\
2
\end{array}\right) .
$$

Proof. Consider a word

$$
\omega=a_{1} a_{2} \cdots a_{s-1} a_{s} a_{s-1} \cdots a_{1} b_{1} b_{2} \cdots b_{s-1} b_{s} b_{s-1} \cdots b_{2} b_{1}
$$

We call such a word fixed doubly palindromic. Now let $\omega_{1} \in \bar{\omega}$. Since $\sigma(\omega)=\phi^{k / 2}(\omega)$, by Lemma 1, all $\omega_{1}$ will have property $\sigma\left(\omega_{1}\right) \in \bar{\omega}$. Furthermore, assume

$$
a_{1} a_{2} \cdots a_{s-1} a_{s} a_{s-1} \cdots a_{1} a_{1} \neq b_{1} b_{2} \cdots b_{s-1} b_{s} b_{s-1} \cdots b_{2} b_{1} .
$$


Then $\omega$ and subsequently $\omega_{1}$ cannot have an even subperiod. By Lemma 2, any such word which is a palindrome must have a subperiod. If a fixed doubly palindromic word is not a palindrome, we can remove its equivalence class from our bound, as reflection of that word would yield a nonidentity cyclic shift of that word. We count the number of non-palindromic classes by counting all fixed doubly palindromic classes and subtracting the fixed doubly palindromic classes with subperiod $d \neq k$. The number of fixed doubly palindromic equivalence classes is established by first counting the number of possible palindromes $a_{1} a_{2} \cdots a_{s-1} a_{s} a_{s-1} \cdots a_{1}$. We know $s=\frac{k+2}{4}$. Thus the number of such palindromes is $n^{(k+2) / 4}$. We then choose two distinct such palindromes to form our equivalence class, giving the total number of equivalence classes as $\left(\begin{array}{c}n^{(k+2) / 4} \\ 2\end{array}\right)$. To count the number of equivalence classes with nontrivial subperiods, we first note that all odd subperiods of length $d$ have the property that $d \mid \frac{k}{2}$. Furthermore, since the equivalence classes with subperiod we are counting form a palindrome, the subperiod word itself must be palindromic. Therefore, the number of possible different subperiods of length $d$ is $\left(\begin{array}{c}n^{(d+1) / 2} \\ 2\end{array}\right)$. The total number of equivalence classes with subperiod, therefore, is $\sum_{d \mid k / 2, d \neq \frac{k}{2}}\left(\begin{array}{c}n^{(d+1) / 2} \\ 2\end{array}\right)$.

Thus, the number of primitive equivalence classes of form $\omega$ is $\left(\begin{array}{c}n^{(k+2) / 4} \\ 2\end{array}\right)-\sum_{d \mid \frac{k}{2}, d \neq \frac{k}{2}}\left(\begin{array}{c}n^{(d+1) / 2} \\ 2\end{array}\right)$. Subtracting from the original bound (1), we complete our proof.

Theorem 6. For $k$ even:

$$
W_{r}(k, n) \leq\left(\frac{1}{k} \sum_{d \mid k} \mu(d) n^{k / d}\right)-\frac{k n^{(k+2) / 2}}{4}+\sum_{i, j \leq \frac{k}{2}, i, j \text { odd }} \frac{\operatorname{gcd}(|i-j|, k) n^{\frac{\operatorname{gcd}(|i-j|, k)+2}{2}}}{4} .
$$

Proof. Consider a word $\omega=a_{1} a_{2} \cdots a_{v} \cdots a_{2} a_{1} b_{1} b_{2} \cdots b_{w} \cdots b_{2} b_{1}$. Note that such a word is doubly palindromic. Clearly $\sigma(\omega) \in \bar{\omega}$. Now consider the equivalence class $\bar{\omega}$. We begin by counting those equivalence classes. We initially observe $v+w=\frac{k+2}{2}$. There are a total of $\frac{k}{2}$ possible values for $v$ (and subsequently $w$ ), since the length of both palindromes $a_{1} a_{2} \cdots a_{v} \cdots a_{2} a_{1}$ and $b_{1} b_{2} \cdots b_{w} \cdots b_{2} b_{1}$ must be odd. This gives $k n^{(k+2) / 2}$. However, this will count both $\omega$ and $\phi^{2 v-1}(\omega)$. Therefore, we divide by two to find our total number of equivalence classes. Thus the total number of such equivalence classes is $\frac{k n^{(k+2) / 2}}{4}$.

However if $\omega_{1}=\omega_{2}$, where

$$
\begin{aligned}
& \omega_{1}=a_{1} a_{2} a_{3} \cdots a_{g} \cdots a_{3} a_{2} a_{1} b_{1} b_{2} b_{3} \cdots b_{h} \cdots b_{3} b_{2} b_{1} \\
& \omega_{2}=c_{1} c_{2} c_{3} \cdots c_{v} \cdots c_{3} c_{2} c_{1} d_{1} d_{2} d_{3} \cdots d_{w} \cdots d_{3} d_{2} d_{1},
\end{aligned}
$$

there is overcounting. By Lemma 4 , such a situation forces $\omega_{1}$ and $\omega_{2}$ to have a subperiod of length $\operatorname{gcd}(|i-j|, k)$ where $i=2 g-1$ and $j=2 v-1$. To count these equivalence classes, we assume without loss of generality that each $i$ and $j$ is at most $\frac{k}{2}$. Furthermore, we note that since we have a word such that $\sigma\left(\omega_{1}\right) \in \bar{\omega}_{1}$, the subperiod must have the same property. By Lemma 3, this means the subperiod must take 
the form $g_{1} g_{2} \cdots g_{r} \cdots g_{2} g_{1} h_{1} h_{2} \cdots h_{t} \cdots h_{2} h_{1}$. We proceed to count all such subperiods using a method similar to that used to count all doubly palindromic words. This yields $\sum_{i, j \leq \frac{k}{2}} \frac{\operatorname{gcd}(|i-j|, k) n^{\frac{\operatorname{gcd}(|i-j|, k)+2}{2}}}{4}$. Furthermore, by Lemma 4, this also counts the total number of words with subperiod in our original count.

We subtract to yield $\frac{k n^{(k+2) / 2}}{4}-\sum_{i, j \leq \frac{k}{2}} \frac{\operatorname{gcd}(|i-j|, k) n^{\frac{\operatorname{gcd}(|i-j|, k)+2}{2}}}{4}$ as the total number of doubly palindromic equivalence classes without subperiods or overcounts. Since each of these classes produces a word whose reflection is also a cyclic shift, none can be contained in a self-reflective comma-free dictionary. Thus we can subtract this number $\omega$ from the original bound (1) to gain our desired result.

\subsection{Applications}

Despite the youth of self-reflective comma-free codes many applications have surfaced. The problem which inspired self-reflective coding is that of efficient use of a receiver. The receiver needs to know fewer words, as it can compare both a string of letters and the reflection of that string to synchronize the code. This is especially useful when a receiver needs to be particularly space-efficient. Furthermore, self-reflective comma-free codes can be used as bijections to a variety of palindromic problems. Apart from the obvious applications for combinatorial problems regarding palindromes, there are a variety of other ramifications. A tight bound on the size of a self-reflective comma-free dictionary when $k$ is even would give a lower bound on the size of a standard comma-free dictionary for even $k$. This is particularly useful, because it bounds a quantity from below which is already bounded from above, and has ramifications for the applications of standard comma-free codes.

\section{Self-swappable comma-free dictionaries}

We define a dictionary $D_{s}$ to be self-swappable if it is fixed under the permutation $f(\omega)=$ $\left(a_{1} a_{2}\right)\left(a_{3} a_{4}\right) \cdots\left(a_{n-1} a_{n}\right)$ where all $a_{i}$ are members of an $n$-letter alphabet where $n$ is even. We denote the maximum number of words a self-swappable comma-free dictionary can contain given $k$-letter words and an $n$-letter alphabet as $W_{s}(k, n)$.

Lemma 5. If $\omega \in D_{s}$ and $f(\omega) \in \bar{\omega}$, either $f(\omega)=\phi^{k / 2}(\omega)$ or $\omega$ has subperiod $d \neq k$.

Proof. We know the permutation $f(\omega)$ has order 2. Thus if $f(\omega)=\phi^{m}(\omega)$, then $\omega=$ $\phi^{2 m}(\omega)$. In other words, such a word must be fixed under a cyclic shift of size $2 m$. It follows that either $k=2 m$ or the word has some subperiod $d \neq k$ (as any word fixed under a nonidentity cyclic shift is not primitive). This observation completes the proof. 
Theorem 7. For $n$ and $k$ even,

$$
W_{s}(k, n) \leq \frac{1}{k} \sum_{d \mid k} \mu(d) n^{k / d}-\frac{1}{k}\left(n^{k / 2}-\sum_{d \mid k, k / d \text { odd }} n^{d / 2}\right)
$$

Proof. To determine this bound, we remove the number of equivalence classes $\bar{\omega}$ satisfying $f(\omega) \in \bar{\omega}$ from bound (1). We remove these, because for all words $\omega \in D_{s}, f(\omega) \in$ $D_{s}$. Since $f(\omega)$ is a cyclic shift of $\omega$, we remove the equivalence class. We count the size of the equivalence class by first counting the number of words $\omega_{1}$ which have the property that $f\left(\omega_{1}\right)=\phi^{k / 2}\left(\omega_{1}\right)$. This number is found by constructing words $\omega_{1}=$ $a_{1} a_{2} a_{3} \cdots a_{k / 2} b_{1} b_{2} b_{3} \cdots b_{k / 2}$ where permutation $f$ takes all $a_{i}$ to all respective $b_{i}$. The number of such words is $n^{k / 2}$. We then subtract the number of words $\omega_{1}$ which have subperiod $d \neq k$. We know $k / d$ cannot be even, because that would require all $a_{i}$ and $b_{i}$ be equal, which is never true. This means $k / d$ is odd. Furthermore, since $k / d$ is odd, the subperiod must take the form $a_{k / 2-d} \cdots a_{k / 2-1} a_{k / 2} b_{1} b_{2} \cdots b_{d}$. Furthermore, the first half of the subperiod in this section must be the same as the first half of the subperiod starting the word. Thus the subperiod must take the form $a_{1} a_{2} \cdots a_{d} b_{1} b_{2} \cdots b_{d}$. This means we

can count the subperiod by $\sum_{d \mid k, k / d \text { odd }} n^{d / 2}$. We then subtract this from our count of all words of form $\omega_{1}$ and divide by $k$ to count the number of equivalence classes. Subtracting from the original inequality gives our desired bound.

\subsection{A construction for self-swappable comma-free dictionaries of word-length three}

We consider the original construction for dictionaries of word-length 3 given by Crick, Griffith, and Orgel [2]. We slightly modify this original construction to create a selfswappable dictionary. In this construction, $A B{ }_{B}^{A}$ represents $\mathrm{ABA}$ and $\mathrm{ABB}$ and the numbers 1 through $n$ represent an $n$-letter alphabet.

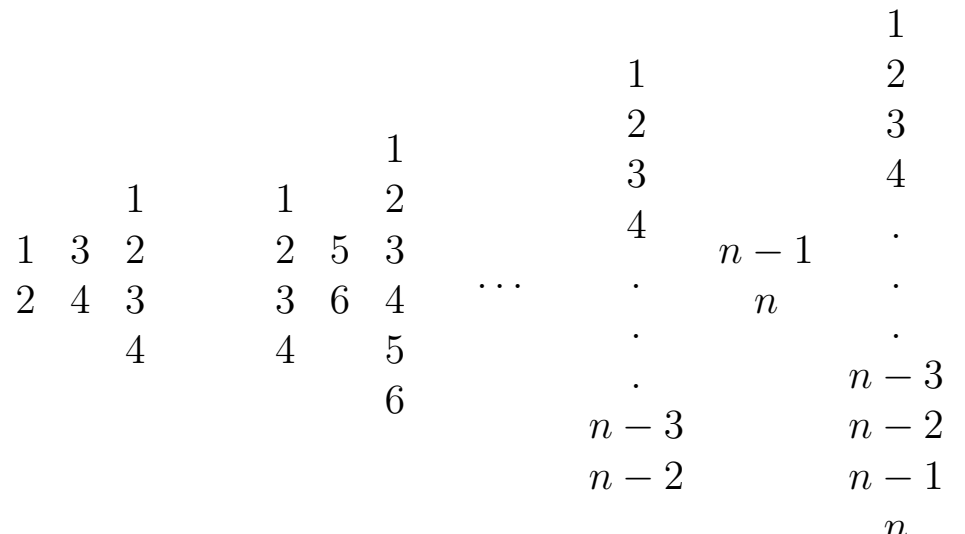

This construction is comma-free and self-swappable. It gives a total of $\frac{n^{3}-4 n}{3}$ words over an $n$-letter dictionary. This differs by the bound for standard comma-free code 
dictionaries of size 3 by exactly $n$ from bound (1) which for $k=3$ is $\frac{n^{3}-n}{3}$. An improved construction or proof of tighter bound is an open problem.

\section{Comma-free matrices and q-dimensional comma- free codes}

Now consider a new type of problem in which we define a comma-free matrix dictionary $D^{2}$ as a set containing matrices with dimensions $k_{1}$ by $k_{2}$ which have the property that for any arrangement of matrices from $D^{2}$ on a plane, any "overlaps" are not in $D^{2}$. That is to say, any $k_{1}$ by $k_{2}$ array chosen in a plane of letters created by words from $D^{2}$ is not in $D^{2}$. We extend the problem to any $q$-dimensional array of letters. We denote a $q$-dimensional comma-free dictionary as $D^{q}$. The maximum number of words such a dictionary can contain over $n$ letters and with word-size of $k_{1} \times k_{2} \times \cdots \times k_{q}$ is denoted as $Q\left(k_{1}, k_{2}, \ldots, k_{q}, n\right)$.

\subsubsection{Möbius inversion for multivariant expressions}

Before establishing bounds for comma-free dictionaries in multiple dimensions, we must establish Möbius inversion for multivariant expressions. Note that summing over multiple variables in the Möbius inversion formula

Lemma 6. $\sum_{d_{i} \mid k_{i}} f\left(d_{1}, d_{2}, \ldots, d_{q}\right)=g\left(k_{1}, k_{2}, \ldots, k_{q}\right)$ is equivalent to

$$
f\left(k_{1}, k_{2}, \ldots, k_{q}\right)=\sum_{d_{i} \mid k_{i}}\left[\left(\prod_{i=1}^{q} \mu\left(k_{i} / d_{i}\right)\right) g\left(d_{1}, d_{2}, \ldots, d_{k}\right)\right]
$$

Now that we have this formulation, we can proceed to our general bound for commafree codes in multiple dimensions.

\section{Theorem 8.}

$$
Q\left(k_{1}, k_{2}, \ldots, k_{q}, n\right) \leq \frac{\sum_{d_{i} \mid k_{i}}\left[\left(\prod_{i=1}^{q} \mu\left(k_{i} / d_{i}\right)\right)\left(\prod_{i=1}^{q} d_{i}\right)\right]}{\prod k_{i}}
$$

Proof. We define a word with subperiod of size $d_{1} \times d_{2} \times \cdots \times d_{q}$ as a word formed by repeating a word of size $d_{1} \times d_{2} \times \cdots \times d_{q}$ to form a word of size $k_{1} \times k_{2} \times \cdots \times k_{q}$. We note that a word must have a subperiod of size $k_{1} \times k_{2} \times \cdots \times k_{q}$ to be in a comma-free dictionary. Otherwise, placing the word next to $2^{q}$ copies of itself yields the original word as an overlap. 
Let $f\left(d_{1}, d_{2}, \ldots, d_{q}\right)$ be the number of words with subperiod of size $d_{1} \times d_{2} \times \cdots \times d_{q}$. All words of size $k_{1} \times k_{2} \times \cdots \times k_{q}$ must have some subperiod of size $d_{1} \times d_{2} \times \cdots \times d_{q}$ where $d_{i} \mid k_{i}$ for all $i$. The total number of words of size $k_{1} \times k_{2} \times \cdots \times k_{q}$ is $\prod_{i=1}^{q} k_{i}$. Thus,

$$
\sum_{d_{i} \mid k_{i}} f\left(d_{1}, d_{2}, \ldots, d_{q}\right)=\prod_{i=1}^{q} k_{i}
$$

Using our formula for Möbius inversion for multivariant functions,

$$
f\left(k_{1}, k_{2}, \ldots, k_{q}\right)=\sum_{d_{i} \mid k_{i}}\left[\left(\prod_{i=1}^{q} \mu\left(k_{i} / d_{i}\right)\right)\left(\prod_{i=1}^{q} d_{i}\right)\right] .
$$

Furthermore, we create equivalence classes of words which are equivalent under one or more cyclic shifts along any dimension. No two equivalent words can be in a comma-free dictionary, as repeating one word yields all equivalent words as an overlap. There are $\prod_{i=1}^{q} k_{i}$ words in each equivalence class. Thus we can divide by $\prod_{i=1}^{q} k_{i}$ to yield the maximum number of such words that can inhabit a comma-free dictionary. This gives us our desired result.

\subsection{Possible additional bounds}

The bounds determined for $q$ dimensions are not always tight. Indeed, there are several other cases which can be eliminated, though they are more difficult to classify. Specifically, it is possible to eliminate all words which are fixed under some nonidentity cyclic shift over $q$ dimensions. This includes but is not limited to cyclic shifts along a single dimension. Subperiods can take place over multiple dimensions. For instance, in two dimensions, cyclic shifts of a repeated block of letters can yield a subperiod in two dimensions, as in the following example: $\begin{array}{lllll}a_{1} & a_{2} & a_{3} & a_{4} \\ a_{3} & a_{4} & a_{1} & a_{2}\end{array}$. Since such matrices cannot be comma-free, they can improve existing bounds; however, their properties are inconsistent. This makes a tight bound difficult. For this reason, we have not utilized this observation to improve our bounds.

\subsection{Self-reflectivity in multiple dimensions: implications and applications}

Now we combine two original concepts in this paper: self-reflective comma-freeness and comma-free codes in multiple dimensions. We expand our definition of words in multiple dimensions to include arrays on a multidimensional lattice of size $k_{1} \times k_{2} \times \cdots \times k_{q}$ with orientation along any dimensional axis. We define a Multiorientational Comma-Free 
Dictionary by requiring that if a multidimensional word $\omega$ is in our dictionary, so too must be all dimensional orientations of $\omega$. Since there are $q$ dimensions, there are thus $2^{q}$ words which are all possible orientations of any word. Since standard self-reflective comma-free codes are in one dimension, there are two orientations of any word: forward and backward. In other words, for each word in a multiorientational dictionary, its reflection must also be in that dictionary. Thus self-reflective comma-free dictionaries are the special case of multiorientational comma-free dictionaries for one dimension.

The implications of multiorientational comma-free dictionaries are staggering. By utilizing a single dimension for a standard word and filling the rest of a multidimensional word with a uniform extra character, it is possible to create a variable-size comma-free dictionary, as the size of a word in each dimension can contain as many as $q$ different lengths in $q$ directions. Variable-size comma-free dictionaries have even more surprising applications. Variable-size comma-free dictionaries have direct implications to the NPcomplete Post Correspondence problem. If all words in the Post Correspondence problem were members of some variable-size comma-free dictionary, the problem would have no solutions. As this has implications to an undecidable decision problem, variable-size comma-free dictionaries have enormous implications in theoretical math. Comma-free codes in multiple dimensions also display potential for future coding and cryptographic techniques.

\section{Conclusion}

This work addresses the new problem of self-reflective comma-free codes. These codes address the critical problem of efficient use of stamp printing by a receiver. This work attempts to gain bounds on the size of self-reflective comma-free dictionaries given variable word-length and alphabet size. This work also discusses the new problem of self-swappable comma-free codes and the generalization to comma-free codes in multiple dimensions.

We achieve tight bounds for specific word-length and variable alphabet length, as well as general bounds for general word-length. The results are limited in scope to constructions under which a reflection is equivalent to a cyclic shift. We proceed to address other classes of comma-free codes including self-swappable codes and comma-free codes over $q$ dimensions. We prove general bounds for these classes, but they contain many open problems. Future extensions of this project could include attempts at tight bounds for general word-length as well as efficient methods of construction for self-reflective commafree codes. Improved bounds on comma-free codes in multiple dimensions should also be attempted. 


\section{Acknowledgements:}

I would like to thank the Research Science Institute in coordination with the Massachusetts Institute of Technology for the opportunity to perform this research over Summer 2007. Additionally, I would like to thank Ms Amanda Epping Redlich, of MIT, for mentoring me and suggesting Comma-Free Codes as a field of study in coordination with Dr P. W. Shor and Dr David Jerison. Finally, I would like to thank Dr John Rickert for teaching me how to write a research paper and Harrison Chen and Allison Gilmore for helping to proofread the paper.

\section{References}

[1] K. L. Collins, P. W. Shor, and J. R. Stembridge. A Lower Bound for 0, 1, * Tournament Codes. Discrete Math. 63, (1987) 15-19.

[2] F. H. C. Crick, J. S. Griffith, and L. E. Orgel. Codes Without Commas. Proc. Nat. Acad. Sci. 43 (1957), 416-421.

[3] W. L. Eastman. On the Construction of Comma-Free Codes. IEEE Trans. Inform. Theory 11 (1965), 263-266.

[4] S. W. Golomb, B. Gordon, and L. R. Welch. Comma-Free Codes. Canad. J. of Math. 10 (1958), 202-209.

[5] B. H. Jiggs. Recent Results in Comma-Free Codes. Canad. J. Math. 15 (1963), 178187.

[6] V. I. Levenshtein. Combinatorial Problems Motivated by Comma-Free Codes. J. Combin. Des. 12 (2004) 184-196.

[7] R. A. Scholtz. Maximal and Variable Word-Length Comma-Free Codes. IEEE Trans. Inform. Theory 15 (1969), 300-306. 


\section{Appendices}

\section{A Constructions for self-reflective comma-free codes}

\section{A.1 Self-reflective comma-free codes of word-length 4}

We construct a self-reflective comma-free dictionary for $k=4$ by including all words ABCD such that $A>B>C \leq D$ or $A \geq B<C<D$. This construction is self-reflective and comma-free. The size of the dictionary created by the construction over an $n$-letter alphabet is $\frac{n^{4}-2 n^{3}-n^{2}+2 n}{4}$. The bound from theorem 6 on the size of such a dictionary is $\frac{n^{4}-2 n^{3}+n^{2}}{4}$. This differs from the size of the construction by $\left(\begin{array}{l}n \\ 2\end{array}\right)$. It is interesting to note the size of the construction for $n=4$. According to Levenshtein [6], the maximum size of a comma-free dictionary with $k=4$ and $n=4$ is 57 . This is 3 less than bound (1) would predict. The size for a self-reflective comma-free dictionary under this construction for $n=4$ is 30 . This is 6 less than the bound from Theorem 6 would predict. It is possible that for each of the three words which could not fit into the 60-member dictionary, those words and their reflections must be eliminated from a self-reflective dictionary, yielding 30 words.

\section{A.2 Self-reflective comma-free codes of odd word length}

We construct a self-reflective comma-free dictionary for odd $k$ by including all words $a_{1} a_{2} \cdots a_{k}$ such that

$$
a_{1}>a_{2}>\cdots>a_{t}<a_{t+1}<\cdots<a_{k}
$$

This construction is self-reflective and comma-free, but it is not a maximal construction for all $k$. Despite this, it is a convenient and consistent method of construction for selfreflective comma-free dictionaries. 


\section{B Other comma-free conjectures}

\section{B.1 Creating new dictionaries from existing dictionaries}

One conjecture we addressed was the potential that for every comma-free dictionary $D=$ $\omega_{1}, \omega_{2}, \ldots, \omega_{x}$, there exists another comma-free dictionary $D^{\prime}=\phi\left(\omega_{1}\right), \phi\left(\omega_{2}\right), \ldots, \phi\left(\omega_{x}\right)$ created by taking a cyclic shift of each word in the dictionary. This is not necessarily true.

Without loss of generality, let $a_{1} a_{2} \cdots a_{k} \in D$ and $b_{1} b_{2} \cdots b_{k} \in D$. Now $D^{\prime}$ must contain $a_{2} a_{3} \cdots a_{k} a_{1}$ and $b_{2} b_{3} \cdots b_{k} b_{1}$, so $D^{\prime}$ cannot contain any of $a_{3} a_{4} \cdots a_{k} a_{1} b_{2}, a_{4} a_{5} \cdots a_{k} a_{1} b_{2} b_{3}$, $\ldots, a_{1} b_{2} b_{3} \cdots b_{k}$.

Thus $D$ could not have contained any of $b_{2} a_{3} a_{4} \cdots a_{k} a_{1}, b_{3} a_{4} a_{5} \cdots a_{k} a_{1} b_{2}$, and so on.

But it may be feasible to include some of these words in D, since they are not necessarily overlaps of the original two words. Thus if $D$ is comma-free, $D^{\prime}$ is not necessarily commafree.

\section{B.2 Creating new dictionaries from existing dictionaries using half-shifts}

While it is not possible to create new dictionaries from any cyclic shift of every word in the dictionary, it is possible to create new dictionaries using cyclic shifts of $\frac{k}{2}$ provided $k$ is even. This is clear, because it is possible to consider each string of $\frac{k}{2}$ letters as a single letter over an alphabet of size $n^{k / 2}$. Then a half-shift is equivalent to a reflection over that alphabet. If the original dictionary was comma-free, then this reflection will be comma-free, as the letters formed by words will remain comma-free. 